\title{
LA INDAGACIÓN DIALÓGICA (ID): UNA ESTRATEGIA PARA LA CO-FORMACIÓN DE DOCENTES EN SERVICIO
}

\author{
María Margarita Villegas Graterol ${ }^{*}$ \\ Instituto Tecnológico de Santo Domingo, República Dominicana \\ margaritavillega@hotmail.com \\ Ligia Mercedes Hernández Caamaño** \\ Instituto Tecnológico de Santo Domingo, República Dominicana \\ mechyhernandezc@gmail.com
}

Recibido: 12/09/2016 Aceptado: 25/11/2016

\section{Resumen}

Este estudio, cuyo objetivo fue analizar la efectividad de la Indagación Dialógica (ID) como modalidad de co-formación permanente de profesores, se realizó con docentes en servicio cursantes de postgrado en una institución de educación superior de la República Dominicana. La ID se asumió como una estrategia de comunicación horizontal, entre profesor acompañante y docente acompañado, quienes dialogan colaborativamente con el fin de encontrar respuestas que les permitan interpretar la práctica, reflexionando sobre la misma. Se realizó un estudio de caso, con seis profesores (tres acompañantes y tres

* Licenciada en Educación Mención Preescolar (UNA -Venezuela, 1987). Maestría en Educación, Mención Orientación (UC-Venezuela, 1993). Doctora en Educación (UC-Venezuela, 2003). Profesora titular Jubilada de la Universidad Pedagógica Experimental Libertador (UPEL Venezuela).

** Maestría en Educación en INTEC (1999) Maestría en Supervisión Educativa (INTEC, 1984). Licenciada en Ciencias de la Educación (UASD, 1979). Educadora del nivel inicial desde 1977. 
acompañados). Los datos se recogieron a través de la observación y la entrevista no estructurada, y se analizaron cualitativamente; los resultados revelan que la efectividad de la estrategia se sustenta tanto en el protocolo que se sigue para su ejecución como en los principios que los profesores acompañantes suscriben desde una relación de horizontalidad entre profesionales que aprenden, permitiéndoles reflexionar sobre el quehacer que envuelve dichas prácticas y no a partir de una visión dicotómica orientada básicamente a focalizar necesidades y fortalezas de la misma.

Palabras Clave: Acompañamiento - Diálogo coloborativo - Reflexión - Protocolo

\section{Abstract}

This communication aims to analyze the dialogic inquiry strategy (ID) as a form of permanent co-training conducted with teachers in service and that also participate in graduate programs in an institution of higher education in the Dominican Republic. The ID was assumed as a strategy of horizontal communication between the accompanist teaching and the accompanied teacher who collaboratively dialogue in order to find answers that will enable them to interpret practice, reflecting collaboratively on it. A case study was conducted with three accompanist teachers and three accompanied teachers. Data were collected through observation and an unstructured interview. These were analyzed qualitatively, and the results show that the effectiveness of the strategy is based on both the protocol development as well as in the principles that accompanist teachers evidence, such as horizontal relationship between professional learning that allow other teachers to reflect on their work involving such practices and not from a dichotomous view that is oriented to focus on needs and strengths from these practices.

Keywords: Accompaniment - Collaborative dialogue - Reflection - Protocol.

\section{Introducción}

Este trabajo es producto de la observación in situ de una estrategia aplicada en el Programa de Acompañamiento Pedagógico gestionada por el Centro de Estudios Educativos del Instituto Tecnológico de Santo Domingo (CEED-INTEC, 2014), que se brinda a todos los estudiantes que cursan los programas de postgrado de especialidades y maestrías coordinados por el CEED. El mismo, se propone desarrollar un proceso de formación y acompañamiento orientado a la autorreflexión, la autonomía profesional, el compromiso y el empoderamiento de los conocimientos y estrategias adquiridas durante los estudios del postgrado.

Una de las actividades que se llevan a cabo es el acompañamiento en contexto, el cual, en el caso de la Especialidad en Educación Inicial del IN- 
Revista de la Escuela de Ciencias de la Educación, año 13, nRo. 12, vol. 1, enero a Junio de 2017. PÁGINAS 97-113. ISSN 1851-6297 - ISSN 2362-3349 (EN LiNEA) LA INDAGACIÓN DIALÓGICA (ID): Una estrategia para la co-FOrmación de docentes en servicio. María M. Villegas Gratero

TEC, es realizado por un grupo de Profesores Acompañantes (PA), dirigido a los profesionales en servicio que están cursando la Especialidad mediante la gestión de estrategias que se ponen en juego durante su quehacer laboral cotidiano. En ellos, las Profesoras Acompañantes (PA) ejecutan acciones para colaborar con las Docentes Acompañadas (DA) en la construcción de un ejercicio profesional adecuado con las demandas contextualmente situadas; tales acciones se realizan con el objeto de "apoyar a los docentes en su labor educativa y promover la formación continua desde y para el centro educativo" (Hernández y Vidal 2014, p.14).

Esta formación continua, desarrollado en el propio centro educativo, busca articular acciones desde diferentes contextos (personal, profesional, hogar, centro y comunidad) de actuación de las DA en función de su propia cultura, con la intención de ayudarles a conferirle sentido a la trama de fenómenos que allí acontecen (INTEC, 2013).

Teniendo esto en cuenta, en la Especialidad en Educación Inicial se asumió el Acompañamiento Pedagógico (Martínez y González, en Hernández y Vidal, 2014, p.18) como una estrategia para impulsar y apoyar procesos de construcción y de formación orientados al desarrollo de culturas socioeducativas recuperadoras e impulsoras de prácticas innovadoras y profesionalizantes. Con las estrategias de acompañamiento en contexto se pretende, así mismo, disminuir la brecha entre la práctica y la teoría que se genera cuando el docente recibe una formación de postgrado fuera de su lugar de trabajo; a ello se le atribuyen los bajos niveles de impacto en la mejora de los aprendizajes en el aula.

El estudio al que se refiere este reporte tuvo como propósito examinar las condiciones de realización de la ID como una estrategia de formación en la cual Profesores Acompañantes (PA) gestionan un diálogo en torno a la práctica docente desarrollada en contexto a fin de promover en la DA la producción de conocimientos sobre su quehacer en las aulas inmediatamente después del haber culminado su clase.

Con base en la información recaudada, se pudo concluir que la ID constituye una estrategia idónea para propiciar que las docentes: a) tomen conciencia del valor de su práctica; b) reconozcan que pueden producir conocimiento pedagógico a partir de una recuperación reflexiva de sus experiencias cotidianas; $y, c)$ comprendan que al compartir vivencias con sus pares pueden enriquecer su quehacer profesional.

\section{Problemática}

La sociedad del conocimiento, caracterizada por la multiculturalidad y la rapidez que imponen las tic's en la producción de saberes, modifica las formas como las personas actúan, piensan y conviven en ese contexto. Así, en el entorno escolar, el quehacer y la carrera docente se ha complejizado, incremen- 
tándose la necesidad de atenuar las distancias entre los saberes producidos por la comunidad científica y la interpretación de los mismos que se ponen en juego en las prácticas del aula, por lo cual la formación de los docentes debe ser continua y permanente.

De allí que hayan emergido los programas de formación continua como una vía para compensar las crecientes necesidades de formación que tienen los profesionales hoy en día; y, de esta manera, mantener actualizados a los docentes en servicio (Posada Álvarez, 2004; Instituto Nacional de Formación Magisterial, INAFOCAM, 2013).

Las insuficiencias de las modalidades tradicionales de formación permanente de docentes han motivado el ensayo de otras estrategias formativas que estén más próximas de las condiciones reales de los centros educativos donde ellos se desempeñan (Vezub y Alliaud, 2012). Actualmente se asume la escuela no solo como una institución donde se ejerce la docencia para otros (los estudiantes), sino que también se la considera como un espacio para la autoformación y co-formación docente. Por ello, el AP ha devenido en una opción idónea para apoyar al docente en servicio en la construcción de saberes desde su práctica.

A nivel nacional, como dificultades se tienen, por un lado, la falta de una perspectiva compartida sobre lo que son las estrategias de acompañamiento $y$, por el otro, la ausencia de un registro sobre los procesos de formación en contexto desarrollados por las instituciones donde los docentes trabajan. Actualmente, se manejan dos posiciones en la práctica de la EAP; una, la concibe como una estrategia para distinguir necesidades y problemas, donde el PA ejerce una función más directiva, haciendo propuestas de soluciones; la otra, plantea que la misma debe realizarse en condiciones propicias para una relación horizontal, en la cual tenga lugar la reflexión compartida de profesionales que se interrogan sobre su práctica (Perrenoud, 2004). Esta última es la que se ha asumido en este trabajo.

En la primera de las posiciones sobre práctica de EAP, el ejercicio del poder es vertical: de una relación entre un profesional experto a un novato, lo cual, actualmente, se ha transformado ante los nuevos modos de funcionamiento de las organizaciones, orientadas a darle poder al usuario, donde prevalecen conceptos, tales como: administración horizontal, autoadministración, equipos autodirigidos y aprendizaje continuo (Capper en Argüelles y Gonczi, 2001, p. 208).

En la segunda, el poder está en función de la autoridad que le otorga el saber (Aubert, García, y Racionero, 2009). Este es redistribuido entre DA y PA. La idea es que cada profesional ejerza su pensamiento crítico y construya de forma consciente las nuevas sendas de su andar en el marco de una relación con otro, con quien al comunicarse se expone y, por ende, se compromete por sí mismo. Pasar de relaciones experto-novato a discusiones críticas en 
las cuales es probable, tal como lo sostiene Argüelles y Gonczi (2001), que el "novato" puede ser capaz de hacer aportes valiosos al "experto", debido a la riqueza que significa reflexionar desde la propia práctica y quien, en un momento, puede comprender e interpretar mejor el fenómeno que se intenta analizar.

Así, la ID (Wells, 1999) es un término que ha sido escogido para interpretar el discurso de una de las Estrategias de AP de los programas de postgrado de la institución formadora, ya que como sus verbos expresan, hace referencia a exploración, búsqueda, investigación para encontrar sentido y significado a las acciones e intenciones de lo que hacemos.

Por su parte, el término dialógico alude a un sistema abierto y autorregulatorio de comunicación que se reconoce como parte de un ecosistema de relaciones humanas, a nivel particular y como totalidad, sustentado en procesos de escuchar, reflexionar y comprender sentidos; $y$, asimismo, el lugar del otro como eje fundamental para la construcción de conocimiento y el desarrollo humano profesional. Es con esta intención que se llevó a cabo este estudio en el cual se puso en juego la ID como estrategia co-formativa de docentes en servicio.

Teniendo en cuenta el panorama anterior, se realizó el presente estudio, el cual tuvo los siguientes objetivos:

1. Analizar la Indagación Dialógica (ID) como estrategia para la co-formación permanente de profesores en servicio.

2. Examinar el protocolo empleado para la realización de la ID, a fin de reconocer condiciones y principios en su ejecución.

\section{Marco Teórico de Referencia}

Los conceptos fundamentales que sirvieron de referencia para este trabajo fueron los siguientes:

Enfoque Histórico Cultural, en el cual se reconoce la sociogénesis de la actividad cognitiva; y el lenguaje como una herramienta esencial para la toma de conciencia a partir de la vivencia y la comunicación en contextos situados (Rodríguez Arocho, 2012). Desde ese enfoque se concibe la psiquis como el fenómeno que deviene de las interacciones en situaciones sociales particulares, como parte de procesos externos (extraindividuales) e internos (intraindividuales), y en cuya trayectoria se desarrolla el pensamiento; fenómeno por medio del cual estas interacciones se reestructuran y se reorganizan en las funciones mentales (Vygotsky, 1996).

Desde esa perspectiva, se valora el medio como escenario fundamental para el enriquecimiento sociocultural; por ende, se rescata de Dewey (1916, p.26) la importancia del medio cuando dice: "El entorno social... es verdaderamente educativo en sus fines en la medida en que el individuo comparta o participe en algún tipo de actividad conjunta...". Es decir, el análisis de la 
ReVista de la Escuela de Ciencias de la EduCACIón, AÑo 13, NRO. 12, Vol. 1, enero a Junio de 2017. PÁGINAS 97-113. ISSN 1851-6297 - ISSN 2362-3349 (EN LINEA) LA INDAGACIÓN DIALÓGIca (ID): Una estrategia para la co-formación de docentes en servicio. María M. Villegas Gratero

experiencia con otros favorece el aprendizaje, pues éste se forja como un proceso individual, pero mediado socialmente.

El aprendizaje colaborativo, procura el intercambio socio-cognitivo entre las personas, promoviendo la motivación a fin de elevar la calidad del conocimiento que se construye conjuntamente (López y Álvarez 2011, p.161). Esta estrategia se sostiene en el trabajo entre personas con niveles de conocimiento similares para el logro de metas comunes (Dillenbourg, 1999; Prendes, 2007). Uno de sus propósitos, en los entornos escolares, es que los actores educativos se capaciten en la autoevaluación y autorregulación de su propia labor educativa (Jugo Cairo, 2014).

Este implica, de acuerdo con Arandia Loroño, Alonso-Olea y Martínez-Domínguez (2010), un proceso que lleva al aprendiz a tomar conciencia real sobre su propio camino de aprendizaje y a consolidar un pensamiento argumentativo y crítico (Rogoff, 1993). Este tiene lugar entre sujetos de conocimiento que se ubican en una situación educativa entendida como acto de conocimiento; no de memorización ni de transmisión, sino de problematización de la realidad que conduce a un análisis y reajuste del conocimiento (Freire, 1990), en la cual el lenguaje se erige como una herramienta vital para el aprendizaje (Bruner, 1975).

El acompañamiento pedagógico, es concebido por Martínez y González (2010, pp. 529-532) como un proceso integrador y humanizador, orientado a abstraer e interpelar las experiencias escolares, como un medio para fortalecer la formación a lo interno de los centros educativos. Por ende, su propósito se orienta a promover la autonomía progresiva del docente y el hábito de la reflexión continua antes, durante y después de su accionar pedagógico (Jugo Cairo, 2014).

La Indagación Dialógica, es tomada de Wells (1999), por concebirse como una herramienta clave en el desarrollo de una actitud y voluntad de exploración, análisis de situaciones educativas con la ayuda de otros, a fin de construir conocimiento situado y contextual para transformar la práctica; admitiéndose, asimismo, como una estrategia de comunicación horizontal (Perrenoud, 2004) entre PA y DA, quienes dialogan colaborativamente con el propósito de encontrar respuestas que les permitan interpretar la práctica, asumiendo una posición reflexiva sobre la misma.

En la sociedad actual, se reconoce al diálogo como un medio idóneo para comunicarnos (Aubert, García, y Racionero, 2009). En el diálogo es posible el reconocimiento del otro que hace el yo, elemento fundamental para el ejercicio de la democracia, sistema político que valora el derecho a disentir y deber de ser tolerante con las diferencias. El diálogo permite que el ser humano perciba su finitud y extensión en el encuentro con el otro, ya que donde empieza uno continúa el otro, el cual a la vez enriquece el yo, propiciando un nosotros.

El logro compartido es producto del diálogo enriquecedor, ya que éste me- 
dia para hacer posible la intersubjetivación de la experiencia y de la comunicación (Flores y Villegas, 2007), haciendo viable la construcción colectiva. Por ende, el diálogo es una herramienta para la construcción de conocimiento compartido, donde se valora la importancia del proceso de formación como un compromiso de autoformación, en el cual cada persona como ser particular se auto dirige y autorganiza para aprender; y como proceso colectivo de co-formación colaborativa y de accionar en un escenario específicamente situado.

\section{Método}

El estudio tuvo carácter exploratorio y en el mismo intervinieron tres parejas, cada una de las cuales estuvo conformadas por una docente estudiante de postgrado de Educación Inicial (DA) y una Profesora Acompañante (PA); el diseño fue un Estudio de Caso de análisis de prácticas en contexto; se utilizaron técnicas de observación no participante, así como entrevistas no estructuradas; la recuperación de la información se realizó a través de la transcripción de las grabaciones de audio, generándose así un corpus que fue sometido a técnicas de análisis de contenido.

Una vez transcripta, la información fue organizada en matrices ad hoc, en las que fueron detectados los identificadores y definidos los indicadores que permitieron construir las categorías que fueron usadas para apreciar la idoneidad de la ID como estrategia adecuada para la formación de docentes en servicio.

\section{Resultados}

Sustentados en los testimonios de la relación entre los pares de PA y DA ya indicadas, se exponen los hallazgos relacionados con el uso de la ID, como estrategia de co-formación del docente en servicio. Estos se organizan en dos aspectos: a) como protocolo de realización; y, b) como estrategia co-formativa.

La ID como protocolo se desarrolla en las seis fases que se exponen a continuación:

Fase I: Crear condiciones: La PA formula la invitación de crear un escenario propicio para el diálogo, reconociendo la importancia del trabajo realizado, a través de la generación de un ambiente de confianza, amabilidad y respeto. Esto se puede ver en el momento cuando la PA realiza la siguiente afirmación: "El aula es como el templo del profesional de la docencia". A través de ello, se está valorando el trabajo de la DA a la vez que focaliza la atención en la dimensión áulica del trabajo. Además, la invita a que exponga sus sentimientos en relación con la presencia de la PA. Esto permite disminuir tensiones y reconocer las emociones que se producen:

PA: ¿Cómo tú estás? ¿Cómo te sentiste con mi presencia en tu salón?

DA: Siente que no estás sola, que alguien te está acompañando. Contigo 
me siento como en más confianza.

Fase 2: Comunicar la intención de la actividad: Esta se objetiva en la siguiente expresión: "que podamos aprender más de cómo tú haces tu trabajo y en lo que podamos ayudarte, también ayudarte". Cuando le dice "podamos aprender" está enfatizando la relación horizontal que ha de existir entre la PA y la DA para encontrar sentido a lo que esta hace.

Fase 3: Focalizar la atención sobre una situación particular observada. Esto es posible a través de la descripción de los hechos alrededor del objeto de atención:

PA: Cuando iniciamos, vimos que trabajaste con unas tarjetas, y que ya habías trabajado la asistencia, pero usaste unas tarjetas para identificar a los alumnos ausentes.

Es decir, promueve la mirada, la observación sobre un asunto particular, haciendo una caracterización del contexto en que ocurrió el evento de interés.

Fase 4: Construir una síntesis de los aprendizajes obtenidos en la ID. En este particular, se le invita a quienes son acompañadas a reflexionar sobre el impacto de la interacción y del mirar la práctica mediada por el diálogo con la PA.

PA: ¿Qué cosas crees que podrías cambiar de ésta, a partir de estos diálogos que hemos tenido? ¿Qué cosas te preocupan y qué crees que puedes mejorar o qué puedes hacer diferente?

Fase 5: Expresar las expectativas para próximo encuentro para ir cerrando la ID y a fin de extender el compromiso de acompañamiento en el tiempo escolar requerido que resta del postgrado y en el ejercicio permanente en el aula:

PA2: A propósito, y con esta reflexión que hemos hecho, ¿que tú piensas que puedes hacer mejor de lo que hiciste hoy?

DA2: Ayudar a esos niños. Hubo un momento en lo que me fui y ya (... ). Ese grupo pequeñito quedó sin atender (...). Yo lo voy a realizar mejor.

PA3: ¿Cómo te gustaría a ti que yo te apoyara a partir de esas necesidades?

DA3: Bueno, me gustarían unos talleres de recursos didácticos, como puedes ver, no soy muy buena con los recursos. Por eso pido ayuda.

PA3: ¿Algo más que tú quieras pedir o que quieras comunicar?

Fase 6: Solicitar retroinformación sobre cómo condujo el proceso de ID. Como se puede observar en la data a continuación, la PA solicita ser retro-informada. A través de ello, la PA muestra actitud de respeto y evidencia que quiere aprender del otro. 
PA2: ¿Cómo te sentiste con esta ID y las preguntas cómo te resultaron?

PA2: ¿Cómo tú me viste como coordinadora haciendo esta indagación?

PA3: ¿Y tú qué piensas de la pregunta y la forma como yo abordé la ID? ¿Cómo fue mi expresión, fue formativa, de ayuda, favorece no favorece? ¿Cómo tú piensas?

PA2: Favorece; favorece porque usted se va a la pregunta de acuerdo al comportamiento (sin cuestionamiento), y no fue una pregunta de arrogancia, sino de lo que vio usted en ese momento.

Como se puede apreciar, el protocolo de acompañamiento empieza creando condiciones para que se inicie el diálogo de manera horizontal; luego, continúa comunicando la intención de la actividad; focalizando la mirada en el fenómeno de interés para la ID; pasando posteriormente, a construir una síntesis sobre el proceso de AP para hacerse conscientes de aprendizajes derivados de la ID sostenida, y con la intención de promover compromiso de reflexión y mejora. Finalmente, para ir cerrando, se solicita comunicar las expectativas para el próximo encuentro y se retro-informe sobre cómo observó el proceso de ID.

\section{ID como estrategia co-formativa}

Cuando hacemos referencia a la ID como estrategia co-formativa, indicamos que nos formamos conjuntamente en la relación con otros, sustentada en el diálogo reflexivo sobre lo que se hace. Por ende, se transforma en una estrategia de colaboración para el aprendizaje de quienes se encuentran con el fin de intercambiar pareceres sobre determinado hecho o fenómeno. Así, en el contexto de este estudio, los PA y las DA se vinculan mediante interacciones verbales, a través de los cuales construyen y reconstruyen los significados y sentidos que le otorgan a determinados procesos y fenómenos didácticos. Algunos de los aspectos encontrados fueron los siguientes:

Refuerzo a la identidad docente. Esto es un elemento fundamental hoy en día, donde se concibe la identidad en proceso de construcción permanente (Matus Rodríguez, 2012), debido a la complejidad de las demandas que se gestan continuamente en el medio social donde se actúa. Por ende, reconocer al otro es vital, robusteciéndose con ello, uno de los principios del aprendizaje dialógico, tal como diálogo igualitario, en el cual se valoran los aportes y el aprendizaje que se consiguen en la relación (Aubert, García, y Racionero, 2009). En este caso, se parte del reconocimiento de las cualidades que se observan en las DA.

PA: El aula es como el templo del profesional de la docencia y te agradece- 
mos el permitirnos estar contigo.

PA: Yo quiero felicitarle. Me gustó que los niños eran muy autónomos. Eso es algo que no se logra de la noche a la mañana. Se ve que lo has venido trabajando desde el primer día de clase.

Toma de conciencia de la intencionalidad didáctica a través de preguntas dirigidas a comprender la relación dinámica de las palabras, las acciones y el pensamiento con el propósito de reconocer las creencias y sus presupuestos epistemológicos (Casilla, Camacho, Finol de Franco, 2008: 287). En el texto siguiente se puede distinguir que la PA sitúa la actividad objeto de análisis, describiendo el contexto en que se dio, para luego formular la pregunta:

PA2: Yo pude observar que tú estabas haciendo la fecha con los niños y le preguntaste qué día era hoy. ¿Cuál era tu intención de preguntarles qué día es hoy?

PA: ¿Cuáles competencias tú tratas de lograr que los niños adquieran haciendo esa actividad?

PA3: Vi cuando los niños estaban trabajando con los nombres, ahí yo quiero felicitarte porque veo que tienes buena memoria porque aún sin el registro, vi que llamaste por su nombre y apellido a cada niño. ¿Verdad que sí? Entonces, jeh!, ¿cuáles competencias tú promueves con los niños en el tarjetero?

Argumentos a favor de la intención didáctica. En este texto se percibe la explicación de la DA1 y 2 sobre lo que persigue, además de revelar sus posiciones teóricas y didácticas sobre cómo aprenden los niños y los contenidos disciplinares objeto de abordaje.

DA1: Con esa estrategia yo utilizo la competencia del pensamiento lógico matemático donde ellos van asociando la cantidad...

DA2: Es para que sepan cuál es la fecha, también para que se ubiquen en el contexto que estamos. Puedes verlo cuando le coloqué el nombre del centro educativo. Y también así, podemos darle los números. Ahí podemos ver el número tres al poner la fecha.

DA1: Bueno, en la competencia comunicativa ellos logran identificar sus nombres y también el nombre de sus compañeros.

De sus respuestas se puede inferir que la DA va haciendo conciencia a medida que va elaborando las respuestas. Esto puede favorecer que a futuro se cuestione el propósito a la luz del currículo con qué objeto hace tal o cual actividad.

Lo invita a revisar otras intenciones. Cuando se observa que la DA 
mantiene una lectura más superficial de la actividad que realiza, se le convoca a pensar sobre otras opciones, a fin de ampliar la perspectiva, la cual se observa restringida a una posición teórica instrumentalista. Así le dice: "PA: cuando tu trabajas con nombres solo promueves la competencia comunicativa, ¿no crees que podemos promover otras competencias?”.

Luego, en otra escena, la PA describe el fenómeno, recupera el contexto a fin de focalizar el objeto de análisis y poder demandar una explicación sobre los fines didácticos. Así lo vemos en el siguiente diálogo:

PA3: ¿Sabes?, a mí me encantó que los niños pudieran cantar. Es bueno cantar, pero que no se queden ahí, que no es cantar solo con el tono de VOZ...

PA3: Yo pude observar que hay unos niños acá que no participaron y realmente no vi lo que tu hiciste, ni vi que hiciste nada para integrarlos y se mantuvieron como muy alejados de la actividad. ¿Qué tú estabas haciendo, sobre todo, cuando recuperaste los aprendizajes previos? ¿Qué pasó ahi?

DA3. Lo noté, pero tú sabes que no es lo mismo estar solo que estar acompañado; luego, a mí se me pasó, como estaba acompañada. Y se me fue, por el momento. Pero sé que debo ayudarlos más para que trabajen igual que los otros niños

También la estimula a ampliar la mirada sobre la actividad que realiza y de cómo la relaciona o conecta con otras disciplinas, en este caso el Pensamiento Matemático y el Proyecto de Agua Saludable.

PA2: ¿Cuál es la relación entre esa actividad del experimento y esta de pensamiento lógico matemático?

DA2: En el grupo pequeño lo que estábamos era identificando números.

PA2: ¿Pero nada que ver con tu experimento?

DA2: No.

PA4. ¡Mmmh! ¿Qué más crees tú?

DA3: Pues, alguna cantidad también.

PA4.: Bien, bien. Esa podría ser una actividad de eso, ¿no?

PA4: Tratar de pensar de manera lógica y no solamente, quizás, identificar los números. Que reconozcan los números ordinales, que desarrollen la capacidad de conectar un conocimiento con otro, que desarrollen la capacidad de observación, que desarrollen la capacidad de inferencia, de establecer hipótesis. Yo creo que es importante ¿no? 
Estimula la reflexión sobre lo que hace, invitándole a revisar el concepto usado, pero asumiendo, primero, que es una lectura personal, principio de respeto y análisis colaborativo, segundo, propiciando la reflexión sobre la afectividad de la estrategia en función de la intencionalidad didáctica.

PA: Me pareció estupendo como trabajas con 32 niños que asistieron hoy ¿Qué quieres lograr reconociendo las normas?

DA: Ver cómo guiarlos, para guiarlos y saber cuáles cosas pueden hacer, en qué momento lo deben hacer; o sea, para eso es la norma, no para prohibirle.

PA: ¿Tú crees que esa frase de tiempo para pensar era la más adecuada para hacerle ver que no acató la norma?

PA: ...Yo diría que revisemos eso. Piensa sustituirlo con otra, con los niños mismos y discutir qué palabra puede ser más adecuada, porque a mí me preocupó...

Se reconocen que los sentimientos están presentes en toda interacción. Por ende, invita a comunicarlos. El propósito es darle permiso para ser disidente, si es el caso. A ser él o ella misma. A expresar con libertad sus sentimientos, los cuales no serán cuestionados. La intención es posibilitar y reforzar la satisfacción laboral, tan importante para el ejercicio de las funciones, producto de sentir que hay un aprecio por el trabajo realizado (Villanueva-Gutiérrez, Tena-Flores y Jaik-Dipp, 2010; Locke, 1976) y la conciencia de que toda actividad está cargada de sentimientos.

PA2: ¿Qué sentimiento te despierta el hecho de que alguien entre a observarte en tu clase?

PA2: ¿Cómo te sentiste con la observación en el aula?

Abordando las inconsistencias. También cuando se observan inconsistencias, por parte de la DA, se le solicita que suministre información para entender algunas dudas que sobrevienen acerca de los asuntos observados. Así está el caso con la DA3 quien realizó un proyecto, pero no se apreciaba que lo había trabajado adecuadamente con los niños. A continuación, diálogo entre PA3 y DA3:

PA3: Muy bien, tú estabas trabajando un proyecto. Vimos un proyecto muy bonito, pero, ¡eh!, ¿Cómo surgió ese proyecto?

DA3: Ese proyecto lo propusieron los niños. En realidad, fue en la semana de transición, cuando estábamos preparando el ambiente para el proyecto. Siempre se ponen libros y revistas, y otra serie de cosas; y ese proyecto partió de un niño, y de ahí le siguieron algunos con varios temas, pero quedó ese.

PA3 ¿Tú crees que en el aula hay evidencia de que ese proyecto surgió de 
los niños?

DA3: ¿En el aula?, yo creo que sí.

PA1: ¿Cuántos votos crees que tuvo?

DA3: Tuvo varios. No recuerdo ahora cuántos votos. Pero tuvo varios. Pero también hemos trabajado varias cosas en relación con el agua...

Los anteriores testimonios revelan que la docente o no sabe cómo es la metodología acordada para escoger un proyecto de trabajo con los niños o no entra en reflexión. Se resiste justificando lo que no realizó. En este caso, la PA3 le hace varias preguntas para hacerla quedar en evidencia de que no se usó la metodología convenida para seleccionar esa actividad, pero la DA3 se acoraza. Y la PA no continúa cuestionándola, pues como reconoce que se está resistiendo hay que dejarla tranquila y darle tiempo para la toma de conciencia.

Por su parte, a la DA2 también se le solicita explicar una situación de inadecuación, pero la actitud de la DA2 es diferente a la anterior (DA3). Se muestra receptiva. Observemos el intercambio verbal:

PA2: Yo pude observar que hay unos niños acá que no participaron y realmente no vi lo que tú hiciste ni vi qué hiciste nada para integrarlos y se mantuvieron como muy alejados de la actividad. ¿Qué tú estabas haciendo?, sobre todo cuando recuperaste los aprendizajes previos. ¿Qué pasó ahí?

DA2: Lo noté, pero tú sabes que no es lo mismo estar solo que estar acompañado; luego a mí se me pasó, como estaba acompañada, y se me fue por el momento. Pero sé que debo ayudarlos más para que trabajen igual que los otros niños.

Invita a recuperar los aprendizajes de la experiencia de la ID. En ese sentido, se observa que se promueve el sentido de colaboración, de construir juntos, de sumar sus ideas, sobre la importancia de trabajar en equipo:

PA1: ¿Qué cosas crees que podrías cambiar de esta experiencia, de estos diálogos que hemos tenido? ¿Qué cosas te preocupan y crees qué puedes mejorar o qué puedes hacer diferente?

DA3: Bueno, como la sugerencia del orden que tengo. Ya sé que para el próximo año voy a ponerlos en orden diferentes. Le voy a agregar los objetivos, también voy a tratar de relacionarlos de manera más concreta, que no me quede solamente en que ellos conozcan la letra, sino que podamos ir más allá con las palabras e igual con todos los nombres que trabajemos.

Este caso permite prestar atención a la importancia del diálogo para com- 
prenderse a sí mismo, lo cual coadyuva a la toma de conciencia de las determinaciones propias e intenciones que guían las acciones (Galvani y Pascal, 2011).

Situar el objeto del acompañamiento. Para cerrar la actividad y como un modo de dejar abiertos algunos espacios para acciones futuras, la PA1 le hace explícita la intención de la actividad desarrollada, la cual se sustenta en el compartir las experiencias de aula, para reflexionar y aprender colaborativamente en una relación de respeto profesional.

PA1: antes de cerrar déjenme decirles que el propósito del acompañamiento es justamente compartir las experiencias, de que conversando sobre lo que vemos y hacemos, nosotras podamos vernos también y aprender sin ningún tipo de "sugestión".

La intención es que en este proceso de ID se construya una formación, donde el saber nacido de la reflexión sobre la experiencia es puesto en diálogo crítico con los saberes disciplinarios, académicos y culturales que ya se poseen (Pineau, 1999); siendo ello una experiencia vital para movilizar el cambio de muchas creencias y supuestos arbitrarios sobre los cuales se sostienen las prácticas.

\section{Conclusiones}

La Indagación Dialógica (ID) como instancia de producción de conocimientos a partir de la Práctica se manifiesta como una oportunidad que tienen las docentes de Educación Inicial para construir conocimientos, aprovechando la mediación cognitiva propiciada por la Profesor(a) Acompañante (PA) quien, a través de preguntas adecuadas, estimula en la Docente Acompañada (DA), la reflexión acerca de las prácticas que ella pone en juego durante sus actividades áulicas en pro del aprendizaje de los/las niños/as que asisten a sus aulas; en síntesis, en la ID se manifiestan cuestiones de diversa índole: (a) cognitiva (estimulación de procesos reflexivos para la construcción de las respuestas a las interrogantes formuladas por la PA); (b) epistemológica (aumento de la conciencia de que es posible construir conocimientos pedagógicos a partir del reconocimiento del vínculo existente entre la DA y su práctica); y (c) social (creación de condiciones para el establecimiento de una relación de apoyo entre DA y PA).

La Indagación Dialógica (ID) constituye una oportunidad para la dimensión socio-afectiva de las relaciones humanas. En efecto, la forma como se lleva a cabo la ID, implica la puesta en juego de interacciones caracterizadas por el respeto y la horizontalidad entre dos profesionales (PA y DA) que comparten perspectivas, lo cual da lugar a un clima socio-afectivo que estimula el mutuo crecimiento personal y profesional; para ello es importante que los participantes de la ID desarrollen habilidades para escuchar, preguntar y observar, ya 
Revista de la Escuela de Ciencias de la Educación, año 13, nRo. 12, vol. 1, enero a Junio de 2017. PÁGINAS 97-113. ISSN 1851-6297 - ISSN 2362-3349 (EN LiNEA) LA INDAGACIÓN DIALÓGIca (ID): Una estrategia para la co-formación de docentes en servicio. María M. Villegas Gratero

que estas capacidades les colocan en mejores condiciones para el trabajo colaborativo con sus pares (Vezub y Alliaud, 2012).

La Indagación Dialógica (ID) permite el reconocimiento y la valoración de la identidad del docente en el encuentro respetuoso implícito en la ID, la cual coadyuva a reforzar la identidad del docente puesto que la PA reconoce la importancia de la labor llevada a cabo por la DA, quien así ve exaltada su actividad áulica, concibiéndola como fuente para la producción de conocimientos con base en las acciones que lleva a cabo cotidianamente en su contexto de actuación profesional.

La Indagación Dialógica (ID) devuelve la voz al docente inverso a lo que ocurre con las interacciones tradicionales en que el docente tiene con sus supervisores, generalmente son asimétricas, y sólo escucha; rara vez es escuchado. La intencionalidad formativa de la ID, al contrario, amerita el despliegue de juegos de lenguaje (Vygotsky, 1977) en los que el docente toma la palabra, puesto que es a partir de sus actos de habla que se desarrolla el diálogo con la PA; de esta manera, la voz del docente es reivindicada, tomada en cuenta, y considerada la base de su propio proceso de formación, mediado por los intercambios verbales que sostiene con la Profesora Acompañante.

La Indagación Dialógica (ID) asume la complejidad de la actividad didáctica, teniendo en cuenta que la misma parte del accionar áulico de la DA y en donde la modalidad de formación docente se fundamente en la práctica, en la cual se reconoce que está contextual y situacionalmente condicionada por un escenario complejo que integra aspectos didácticos, actitudinales, motivacionales, cognitivos, y sociales.

\section{Referencias bibliográficas}

- Arandia Logroño, M., Alonso-Olea, M.J. y Martínez-Domínguez, I. (2010). La metodología dialógica en las aulas universitarias. Revista de Educación, 352, 309-329.

- Argüelles, A. y Gonczi, A. (2001). Educación y capacitación basada en normas de competencias: una perspectiva internacional. México: Limusa,

- Aubert, A.; García, C. y Racionero, S. (2009). El aprendizaje dialógico. Cultura y Educación, 21 (2), 129-139.

- Borquez Hernández, G. (2013). Protocolo de prácticas y encuentros reflexivos. Santo Domingo. R.D. CEED- INTEC: Printcorp Servicios Gráficos Corporativos SRL.

- Bruner, J. (1975). The genesis of speech acts. J. of Child Language, 2, 1-19.

- Capper, P. (2001). La competencia en contextos laborales complejos. En: Argüelles, A. y Gonczi, A. (ed.) (2001) Educación y capacitación basada en normas de competencias: una perspectiva internacional. México: Limusa

- Casilla, D., Camacho, H., Finol de Franco, M., (2008). La indagación: una estrategia innovadora para el aprendizaje de procesos de investigación. Laurus 2008, 14 (EneroAbril) [en linea] [Fecha de consulta: 19 de junio de 2016] Disponible en http://www. redalyc.org/articulo.oa?id=76111491014.

- Centro de Estudios Educativos-Instituto Tecnológico de Santo Domingo. (2014). 
Programa de Acompañamiento Pedagógico. Mimeo.

- Dewey, J. (1916). Democracy and education: An introduction to the philosophy of education. Nueva York: Mac-Millan.

- Dillenbourg, P. (1999). What do You Mean by Collaborative Learning? In P. Dillenbourg (Ed.), Collaborative-learning: Cognitive and Computational Approaches. (pp.1-19). Oxford: Elsevier.

- Flores, N. y Villegas, M. (2007). El Sujeto Dialógico en la Pedagogía de la Investigación. En Bolívar, A. y Erlich, F. (ed.) (2007). El Análisis del diálogo. Reflexiones y Estudios. Caracas: Fondo editorial de Humanidades, UCV, 2007-207-218, Venezuela.

- Freire, P. (1990). La naturaleza política de la educación. Cultura, poder y liberación. Madrid: Paidós.

- Galvani, P. (2011a). Estrategias Dialógico-Reflexivas para la Eco-formación. Visión Docente. Con-Ciencia Año X, No. 59 Mayo - Junio 2011. Disponible: http://www.ceuarkos. com/Vision_docente/estrategias59.pdf. Cito: Pp. 9 y 10.

- Hernández Caamaño, M. y Vidal, C. (2013). Plan Institucional de Formación y Acompañamiento. INTEC-CEED. Impreso por Printcorp Servicios Gráficos Corporativos, S.R.L.

- Instituto Nacional de Formación Magisterial (INAFOCAM, 2013). Marco de la formación Continua: Una perspectiva articuladora para una escuela de calidad. Santo Domingo: El autor.

- Jugo Cairo, M. I. (2014). El acompañamiento pedagógico. Protocolo del acompañante pedagógico, del docente coordinador/acompañante y del formador. Impresión. Ministerio de Educación de Perú. Lima: Industria Gráfica MACOLE S.R.L. Disponible en: http:// www.minedu.gob.pe/opyc/files/Protocolodelacompanantepedagogico.pdf

- López, D., y Álvarez, I. (2011). Promover la regulación del comportamiento en tareas de aprendizaje cooperativo en línea a través de la evaluación. RIED. Revista Iberoamericana de Educación a Distancia, 14 (1), 161-183.

- Locke, E. (1976). The nature and causes of job satisfaction.In M. Dunnell (Ed.).Handbook of industrial and organizational psychology (pp. 1297-1349). Chicago, IL: Rand-McNally.

- Martínez, H. y González, S. (2010). Acompañamiento pedagógico y profesionalización docente: sentido y perspectiva. Ciencia y Sociedad, XXXV Julio-septiembre, 521-541.

- Matus Rodríguez, L. G. (2012). La construcción de una identidad docente, ¿un desafío para la política educativa? IX Seminário da Rede Estrado. Políticas Educativas na América Latina: Praxis Docente e Transformação Social. Consulta: http://www. filosofiayeducacion.ucv.cl/wp-content/uploads/2012/12/Grace-Matus-R.pdf.

- Pineau, G. (1999). Expérienced'apprentissageet histoire de vie. En P. Carré\& P. Caspar (Eds.), Traité des sciences et des techniques de la formation (pp. 307-327). París: Dunod

- Posada Álvarez, R. (2004). Formación superior basada en competencias, interdisciplinariedad y trabajo autónomo del estudiante. Revista Iberoamericana de Educación.Consulta: file:///C:/Users/usuario/Downloads/648Posada. PDF

- Perrenoud, P. (2004). Desarrollar la práctica reflexiva en el oficio de enseñar. Barcelona: Graó.

- Prendes, M.P. (2007). Internet aplicado a la educación: estrategias didácticas y metodologías. En J. Cabero (Coord.), Nuevas tecnologías aplicadas a la educación. (pp. 
Revista de la Escuela de Ciencias de la Educación, año 13, nRo. 12, vol. 1, enero a junio de 2017. PÁGINAS 97-113. ISSN 1851-6297 - ISSN 2362-3349 (EN LiNEA) LA INDAGACIÓN DIALÓGIca (ID): Una estrategia para la co-formación de docentes en servicio. María M. Villegas Gratero

205-222). Madrid: McGraw-Hill.

- Rodríguez Arocho, W. (2012). Perfiles y perspectivas del acompañamiento pedagógico. Mirada crítica y claves para el desarrollo de modelo histórico cultural. Santo Domingo. CEED- INTEC.

- Rogoff, B. (1993). Aprendices del pensamiento. El desarrollo cognitivo en el contexto social. Barcelona: Paidós [V.O. Apprenticeship in Thinking: cognitive development in social context. Nueva York: Oxford UniversityPress, 1990].

- Villanueva-Gutiérrez, R.; Tena-Flores, J.A y Jaik-Dipp, A. (2010). Satisfacción laboral y compromiso institucional de los docentes de posgrado.Revista Electrónica Diálogos Educativos 19 (10) 119-130. Consulta: file:///C:/Users/usuario/Downloads/Dialnet-Satisfa ccionLaboralYCompromisolnstitucionalDeLosDo-3294739.pdf.

- Vygotsky, L. (1977). Pensamiento y lenguaje. Buenos Aires: Pléyade.

- Vygotsky, L. (1996). El desarrollo de los procesos psicológicos superiores. Barcelona: Crítica.

- Vezub, L. y Alliaud, A. (2012). El acompañamiento pedagógico como estrategia de apoyo y desarrollo profesional de los docentes noveles. Aportes conceptuales y operativos para un programa de apoyo a los docentes principiantes de Uruguay. MEC-AECID-ANEPOEl.

- Wells, G. (1999). Dialogic inquiry: Toward a sociocultural practice and theory of education. New York: Cambridge UniversityPress. 